Voix et Images

\title{
Claude Gauvreau : du tombeau du père au langage exploréen
}

\section{Jean-Pierre Denis}

Volume 18, numéro 3 (54), printemps 1993

Littérature, folie, altérité

URI : https://id.erudit.org/iderudit/201045ar

DOI : https://doi.org/10.7202/201045ar

Aller au sommaire du numéro

\section{Éditeur(s)}

Université du Québec à Montréal

\section{ISSN}

0318-9201 (imprimé)

1705-933X (numérique)

Découvrir la revue

\section{Citer cet article}

Denis, J.-P. (1993). Claude Gauvreau : du tombeau du père au langage

exploréen. Voix et Images, 18(3), 483-494. https://doi.org/10.7202/201045ar
Résumé de l'article

Résumé

Traversée par la mort, l'oeuvre gauvrienne est aussi soutenue par l'impérieuse volonté de la renverser, d'agresser le mal en ses racines, c'est-à-dire à partir d'un travail sur la langue. À cet égard, le langage 'exploréen " - cette langue qui se tient contre, tout contre le cadavre de la langue maternelle qu'il abolit et relève tout à la fois - pourrait être ce qui se dresse sur la tombe silencieuse du père pour faire jouir la mère, jusque dans la mort... 


\title{
Claude Gauvreau: du tombeau du père au langage exploréen
}

\author{
Jean-Pierre Denis, Montréal
}

Traversée par la mort, l'œuvre gauvrienne est aussi soutenue par l'impérieuse volonté de la renverser, d'agresser le mal en ses racines, c'est-à-dire à partir d'un travail sur la langue. À cet égard, le langage "exploréen .cette langue qui se tient contre, tout contre le cadaure de la langue maternelle qu'il abolit et relève tout à la fois - pourrait être ce qui se dresse sur la tombe silencieuse du père pour faire jouir la mère, jusque dans la mort...

C'est le silence seul qui comprendra le sens de mes paroles.

Aucun homme sur la terre ne comprendra ce que je dirai.

Je suis un marchand de silence. Le miel suffocant entre deux mots tragiques est mon œuvre.

Les hommes vivent entre deux mots.

Les hommes vivent dans le silence.

Les grands explicateurs sont des morts enfants.

[...]

Incompréhensible discours!

Je parle à mon âme, et mon âme seule comprend alors que je ne comprends pas moi-même ${ }^{1}$.

1. Claude Gauvreau, «Nostalgie sourire •, Guvres créatrices complètes, Montréal, Parti pris, coll.. Du chien d'or, 1977 , p. 56. 
Avant d'aborder le continent "exploreen ${ }^{2}$ " du langage chez Gauvreau, je voudrais d'abord faire un détour qui, je l'espère, sera éclairant, du moins suggestif. Ce détour m'est en quelque sorte imposé par la nature même de cette langue étrange qu'est l'exploréen, langue qui se tient contre, tout contre le cadavre de la langue maternelle qu'elle abolit et relève tout à la fois pour en jouir. J'avancerai donc, pour l'instant, la proposition suivante: le langage exploréen est ce qui se dresse sur la tombe silencieuse du père pour faire jouir la mère, jusque dans la mort...

C'est dire le rapport que j'entrevois, d'une part, entre cette langue et la mort, langue comme jouissance dedans la mort; d'autre part, entre cette langue et la mère à qui elle est destinée: celle-là, archaïque, qui occupe tout l'espace imaginaire des premiers textes gauvriens; celle-là encore qui vient clore son ouvre en recueillant ses derniers jappements glossolaliques dans une sorte d'indifférence meurtrière. La surprésence et la. survalorisation de la mère auront sans doute été, chez Gauvreau, ce qui l'aura détourné d'un geste décisif: accomplir sa propre mort, la mort de son propre, l'arrachement d'avec soi-même qui seul permet au poète la conquête de son $A u t r e$, dans et à travers le langage.

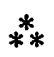

Amorçons donc ce détour par cette citation tirée de Mallarmé: * Pour moi, le cas d'un poète, en cette société qui ne lui permet pas de vivre, c'est le cas d'un homme qui s'isole pour sculpter son propre tombeau ${ }^{3}$."

Par ces mots, Mallarmé rappelle les conditions et le lieu de l'acte poétique au sein de l'époque moderne: l'isolement du poète, son retrait du spectacle enivrant de la vie en faveur des fêtes plus secrètes du langage. Le poète est celui qui maintient, et doit exercer en son cœur, une sourde et secrète résistance au monde, celui qui se tient

2. Le langage "exploréen, s'il est une invention proprement gauvrienne, peut être assimilé à la glossolalie quant à sa forme. On en trouve des exemples à travers toute son cuvre sous forme de soudaines explosions néologiques. Toutefois, seuls quelques textes, comme ceux qui composent Faisceau d'épingles de verre (1961-1970) et Jappements à la lune, sont entièrement exploréens, c'est-à-dire ne font entrer aucun mot connu dans la composition du poème ou de l'objet dramatique.

3. Réponse à une enquête Sur l'évolution littéraire, EEuvres complètes, Paris, Gallimard, coll. «La Pléiade :, 1970, p. 869. 
violemment debout contre son pouvoir d'absorption, contre le trouble de son jour, force dispersante et dé-voyante. Son travail se fait en secret et dans l'austérité, dans le mystère de ce qu'il ne sait pas mais qui insiste en lui. Il exige la plus haute solitude. Arrivé à hauteur de page blanche, à l'orée du dire encore bordé de silence, c'est enfin à la mort qu'il doit consentir, c'est dans la mort qu'il doit se décider à entrer. Là, tout sujet cesse d'exister: «l'œuvre implique - dit Mallarmé - la disparition élocutoire du poète; qui cède l'initiative aux mots ${ }^{4}$. Aboli, donc, le sujet qui croit parler en son nom propre, ainsi que le monde dans sa matérialité qui, selon le jeu du langage, disparaît pour que naisse la "pure notion.. Le poète est ainsi celui qui peut dire: Je suis mort. Ou plutôt: Je est mort.

Le tombeau, c'est la crypte que le poète se construit, qu'il doit ciseler à même le langage - qui est son vrai corps - , car seul à s'assurer cet espace de résonance lui revient, singulièrement densifiée, la parole qui, à travers les siècles, s'est portée jusqu'à lui par la voix d'autres poètes. La crypte, ou l'espace du Livre, l'héritage sonore du temps, sa mémoire, l'exhaussement, enfin, de cette négativité qu'on appelle la mort et qui est déjà dans toute voix en ce que celle-ci est le propre, mais un propre sans commencement, pur mystère d'une origine à soi cachée.

Quand Mallarmé dit que la société ne permet pas à ses poètes de vivre, il faut l'entendre de la bonne oreille. Il ne s'agit pas, ici, de cette réalité qu'ont connue nombre de poètes et d'écrivains québécois, condamnés à s'exiler (intérieurement ou extérieurement), à vivre en l'absence d'eux-mêmes, de leur culture, en manque de monde; parfois même, à vivre leur mort sous la forme, définitive, du suicide. Était-ce parce qu'ils ne pouvaient rencontrer aucun public qui pût les comprendre, aucune histoire qui pût les accueillir, dans le respect et la reconnaissance 5 ? Jacques Ferron, déjà, se plaignait qu'en ce pays on ne respecte pas les morts, que "les morts [ne puissent] reposer en paix, tels qu'ils ont été, en gardant leur identité ${ }^{\sigma_{\text {p }}}$.

Disons simplement ceci: Mallarmé avait, certes, un public, au contraire, par exemple, de Gauvreau; mais en eût-il manqué - je veux dire de lecteurs - qu'il aurait encore éprouvé leur présence, car le premier lecteur, c'est le langage, c'est ce qui est déposé comme

4. Id., Crise du vers, ibid., p. 366.

5. Gauvreau, à la suite de bien d'autres, dira: «[...] le milieu social québécois est le plus ingrat qui soit au monde. (Correspondance, 7 janv. 1950, p. 1). Inédit.

6. Le Devoir, 19 avril 1980, cité par Jean Larose dans La Petite Noirceur, Montréal, Boréal, coll. . Papiers collés *, 1987, p. 63. 
mémoire et comme trace de l'Autre dans la langue. Aussi Mallarmé peut-il écrire: «Impersonnifié, le volume, autant qu'on s'en sépare comme auteur, ne réclame approcbe de lecteur. Tel, sache, entre les accessoires humains, il a lieu tout seul [...] $]^{7}$."

Mallarmé occupait de plain-pied l'histoire de la poésie, des siècles de poésie l'entendaient, il avait sa place depuis toujours dans ce tombeau qu'il a si souvent offert en hommage à la mémoire d'auteurs aimés. Il ne faut donc pas confondre une société qui rend la vie impossible à ses poètes (comme ce fut longtemps le cas au Québec, mais cela a-t-il vraiment changé?), et une société qui ne permet pas au poète de vivre, voire de survivre.

Il est certes difficile pour le poète moderne de continuer à faire entendre sa voix dans une société vouée de plus en plus au journalisme, aux faits divers, impatiente d'agir, agacée par les mystères qu'elle ne sait plus ou ne veut plus entendre que dans la forme, rationalisée, de la science; mais cela va encore dans le sens de ce qu'exige la poésie et de ce qu'elle ordonne au poète: se donner à soimême cette vie par l'épreuve poétique, qui est l'épreuve du sens dans la mort de soi et dans le don au langage. Car il n'y a pas de poésie sans rapport à la mort: le lieu du poème, pour reprendre la formule de Giorgio Agamben, est "toujours un lieu de souvenir et de répétition; le présent de l'instance de discours, un avoir été, un passé". Il n'y a pas de parole pour dire la parole même, le discours ne peut dire son avoir-lieu; c'est pourquoi la parole du poète ne peut être que -l'expérience silencieuse de l'avoir-lieu du langage dans la Voix et dans la mort ${ }^{8}$. Tout poète est confronté à la mort dès qu'il se livre au langage et mesure sa disparition dans le tracé des mots qui avancent, comme malgré lui. Et Gauvreau ne fait pas exception.

La mort fait son entrée dès son premier texte, Les Reflets de la nuit $^{9}$, sous sa forme la plus directe. On y assiste à la suppression de Frédéric Chir de Houppelande, qui se targue d'être le plus grand des poètes, et à son remplacement par une figure inquiétante: Hurbur. Chose singulière, cet Hurbur est présenté par les voix de la forêt comme un "nouveau "Frédéric Chir de Houppelande; en somme, un double négatif du premier. Comme si Gauvreau, dans ce geste inaugural d'écriture, dans ce geste fondateur de sa personnalité d'écrivain, de son style, avait voulu se débarrasser d'une partie de lui-même - trop

7. Stéphane Mallarmé, op. cit., p. 372.

8. Giorgio Agamben, Le Langage et La mort, Paris, Christian Bourgois, 1991, p. 113.

9. Claude Gauvreau, op. cit., p. 19-22. 
tendre, trop romantique, trop liée à la mère sans doute ${ }^{10}$-, au profit d'une personnalité plus dure, plus sadique, plus héroïque. Hurbur sera donc le "maître de la nuit ", de cette même nuit qui hallucinait le premier et le faisait hurler, en proie à des mirages qu'il ne savait déchiffrer.

La jeune fille du début, Corvelle, qui vivait dans les « remous du chant du poète" et dont l'univers entier lui "murmurait dans ses frémissements les paroles bien-aimées de Frédéric Chir de Houppelande ", se tourne dès lors vers son nouveau maître, Hurbur. Il ne faut pas s'en étonner: les femmes, chez Gauvreau, du moins celles qui comptent, sont toujours celles qui. ne peuvent trouver leur voix et avoir un corps que dans la parole du poète. Telles ces hystériques dont parlait Charcot, elles sont en attente du maître qui les façonnera et gravera son verbe en leur chair. Corvelle, donc, lui demande alors de la prendre dans son dos et d'en faire deux "ailes", afin, dit-elle, qu'elles les portent tous deux au ciel. Mais Hurbur, imperturbable, de lui répondre: "Je n'ai pas besoin des ailes, toutes les passerelles aux étoiles me sont soumises, mais tu peux venir avec moi. Je travaille, je travaille sans cesse."

Le travail (l'borrible travail dirait Victor Hugo) contre la tentation dissolvante d'elles? Par le raccourci des passerelles, se passer d'ailes / d'elles? Ou passer par elles?... Tranchons. Il emporte la jeune fille au sein des étoiles, la tue, dans un geste de pure impassibilité, et conclut, laconiquement: "La nuit prend fin."

On a là, dans cette schize initiale et dans ces meurtres à répétition (ou en abyme), tout Gauvreau. La mort traverse en effet l'œuvre gauvrienne de part en part. Elle est ce qui organise récits comme fantasmes, ce qui donne force au désir, ce qui institue, déclenche la quête. Elle est aussi ce qui torture le poète, le met "en souffrance" (comme on dit d'une lettre qu'elle est restée en souffrance, sans réponse). Dans tous les cas, elle est ce qui travaille en profondeur sa langue / ses langues, ce qui trouble le champ de la communication, ce qui rend bègue son énonciation, ce qui insiste et infiltre partout de néant son tissu.

Aborder l'œuvre gauvrienne, c'est être confronté, quasi à chaque page, à l'impérieuse emprise de la mort, et à la non moins impérieuse volonté de renverser le sort, d'agresser le mal en ses racines, de faire

10. Notons au passage le jeu des noms: Frédéric Chir de Houppelande / Hurbur, le premier évoquant la joliesse, la dentelle, la noblesse, pour tout dire l'insignifiance; le second, l'inquiétante étrangeté, l'outre-monde, le lieu caverneux... 
tonner la langue pour effrayer le vide, d'exposer la chair en ses stigmates pour conjurer l'opprobre, de glorifier le corps pour en masquer l'insignifiance, de reprendre inlassablement là où il y a eu perte, mort. Mais c'est aussi, bien souvent, ruser de mauvaise foi avec elle, s'enfoncer dans la méconnaissance en multipliant les doubles, comme en un jeu de poupées gigognes, renoncer au face à face, semer partout la mort pour mieux la maîtriser comme celle de l'autre, ou celle advenant par l'autre. Cette mort, Gauvreau n'en parle pas souvent: il la met plutôt en scène, la théâtralise pour la rendre visible. Pourtant, dans sa correspondance avec Jean-Claude Dussault, il en a parlé à au moins deux reprises. Ce fut, à chaque fois, pour la nier, pour la rendre insignifiante, pour lui enlever tout ascendant sur lui, voire toute réalité:

Pour ma part, la mort me laisse bien indifférent. :L'anxiété des athées. n'est qu'une invention catholique, par laquelle les curés projettent sur les gens sains leur mauvaise humeur envieuse de ne pouvoir copuler à leur goût. (22 mars 1950)

Dans une autre lettre:

Vous me demandiez jadis mes opinions sur la mort. Voici une réflexion. La crainte de la mort est l'un des sentiments les moins justiciables. En effet: le désagrément véritable serait de pouvoir sentir qu'on est mort. Mais, tant qu'on sent quelque chose, on n'est pas mort, et, dès qu'on est mort, on ne sent plus rien. Alors... (10 mai 1950)

Ce n'est certes pas là ce qui s'est écrit de plus lucide sur la mort ou sur l'angoisse de la mort. Et on peut sans doute attribuer en partie cette position à l'optimisme qui régnait à ce moment dans le groupe automatiste, à son parti pris pour la vie concrète, pour la matière, contre l'abstraction et les spéculations métaphysiques, jugées inutiles; contre la mort, aussi, qui régnait en maître dans la société québécoise et étranglait littéralement ses créateurs ${ }^{11}$. Pourtant, c'est bien cette mort, initialement moquée, que nous trouvons partout à l'œuvre dans le texte gauvrien. S'en être débarrassé par un tour de passe-passe, de magie, n'y aura rien changé. La mort sera restée sa maîtresse, et c'est elle qu'il rejoindra dans son suicide.

Pourtant, Gauvreau a souvent donné un nom - sans doute trop concret, trop réel pour être vrai! - à cette mort: Muriel Guilbault. Et on sait, surtout par ses pièces de théâtre et, bien sûr, par son roman

11. - Braquons nos projecteurs sur les prédicateurs à honte perpétuelle

il faut de l'air pur à l'azur mort dans vos mains de fumistes. .

Claude Gauvreau, Les Boucliers mégalomanes 48, op. cit., p. 1247. 
moniste, Beauté baroque ${ }^{12}$, qu'il n'a jamais pardonné à la société d'avoir suicidé sa bien-aimée et, du coup, de l'avoir tué, lui, le poète, l'emmurant à jamais dans cet espace hors monde, hors temps, hors vie, hors amour où il ne lui restait plus qu'à reprendre, inlassablement, ce que la mort, comme figure grimaçante de la société, lui avait ravi.

Sa pièce, $L a$ Reprise ${ }^{13}$, et jusqu'en son titre, est à cet égard tout à fait exemplaire. Elle nous montre le personnage principal (Weddeg, un comédien) occupé jusqu'à l'obsession, jusqu'à la folie à refaire de ses propres mains un double artificiel, un automaton de sa bienaimée (Iritrakk), morte avant d'avoir pu donner la dernière représentation d'une pièce intitulée Le Corps sans larmes. S'enfermant dans son atelier, tel en un tombeau dont il ne veut plus s'arracher avant d'avoir achevé son œuvre, ce compagnon de scène accomplit le miracle de donner cette dernière représentation en compagnie d'un robot, construit de ses propres mains hallucinées, reproduisant exactement (sauf une certaine rigidité, ajoute le narrateur) les gestes de la défunte comédienne. Seule sa voix, n'ayant pu être imitée, devra être rendue grâce à un enregistrement fait avant sa mort. La pièce, comme il se doit, se termine de la manière la plus tragique. Sortant du théâtre, après la représentation, avec le robot dans ses bras, il sera fauché par une voiture; sous la violence du choc, le robot emboutira son corps qui ne fera plus qu'un avec lui, pantin retrouvant l'unité primordiale dans cette déchirante fusion au corps du premier Autre: la mère...

Ce n'est pas le seul texte dramatique où Gauvreau a redonné vie, sous une forme ou sous une autre, à Muriel Guilbault. Ce qui est en revanche symptomatique, c'est l'impossibilité où il s'est trouvé, pendant les vingt ans qu'a duré sa vie de créateur, d'en faire le deuil. Une question se pose donc. S'agit-il bien d'une incapacité propre à l'individu Claude Gauvreau, ou devons-nous considérer plutôt l'auteur, refusant catégoriquement de taire la mort qui est au fondement de notre identité collective comme de notre histoire? Car l'écrivain, le poète, n'est-il pas en tout premier lieu l'analysant de la société?

Gauvreau a passé sa vie à déterrer les morts, et bien d'autres encore que les seuls de sa biographie. Comme si, prenant au pied de la lettre une devise bien connue, Je me souviens, il avait voulu être le témoin brûlant de nos oublis. Son roman, Beauté baroque, ne se termine-t-il pas par un appel à la mémoire, le legs d'une parole testimoniale destinée à une communauté à venir?

12. Ibid., p. 379-500.

13. Ibid., p. 1263-1361. 
-Je suis un témoignage...* - dit Beauté baroque. La parole me suit comme un chien adopté. [...]

Et moi : ne suis-je pas le témoin? se dit le poète.

La mort du témoin est la mort du témoignage ${ }^{14}$.

Cette impossibilité du deuil pourrait donc être le fruit d'une rencontre entre deux histoires: celle d'un sujet biograpbique, Claude Gauvreau - chez qui nous pourrions concevoir cette incapacité à la relation amoureuse très fortement teintée de sadisme qu'il entretenait avec l'imago maternelle, au point qu'il risquait à tout moment de détruire le "moi idéal" qu'il avait placé en elle et dont son immortel corps de gloire dépendait -, et celle d'un auteur qui, repérant à même sa société une situation similaire, crut trouver l'origine de son mal dans ce drame collectif que tous cherchaient à masquer ou à oublier...

Ce drame, à le lire à travers l'œuvre gauvrienne, tourne immanquablement autour de la mort de quelque chose de grand, de sublime, de quelque chose qui aurait été plus fort que la mort si... Si. Comme si Gauvreau avait voulu forcer le Québec à vivre, à travers les diverses catharsis que proposent ses textes, sa propre mort, cette mort perdue, refoulée, déniée au profit d'une vie insipide et sans grandeur, au profit d'un survivre, d'une méconnaissance pire que la mort. Comme s'il avait voulu relever la mort pour la faire apparaître comme mort, pour qu'enfin une reconnaissance collective soit possible.

Dans un de ses premiers textes dramatiques, La Prière pour l'indulgence, on voit, à la fin, le narrateur s'enfermer dans un grand Livre rouge qui a été descendu du plafond avant d'être remonté, emportant avec lui le poète. Ses dernières paroles: "Moi, j’ai trouvé mon cercueil.- Mais... un cercueil, fut-il un grand livre rouge, n'est pas un tombeau, pour reprendre le mot mallarméen: il n'est qu'une boîte dans laquelle on enferme un corps avant de le déposer en terre. Il est ce qui pourrira, comme le corps qu'il renferme, et sans même laisser de traces. Un tombeau, c'est autre chose! C'est un monument, pouvant d'ailleurs servir de sépulture à un ou plusieurs morts; c'est aussi une composition poétique ou une cuvre musicale en l'honneur d'un artiste; d'un grand homme disparu. Le tombeau est pérenne, il est avant tout un symbole efficace, et notamment du NOM qu'il porte; il com-mémore, réunifie la mémoire des successeurs autour d'un nom emblématique, nom débarrassé de sa chair, ayant conquis la dureté et le lisse de la pierre. En somme, il est le "nom du père", il en cèle le secret et témoigne de sa puissance dans la mort. En ce sens, pour reprendre la distinction faite par Heidegger, mourir (sterben) n'est pas décéder (ableben). La mort se gagne, s'arrache au prix d'une mort, d'une perte de ce que la psychanalyse a appelé, dans l'ordre du 
langage, le corps, le soma - qui est aussi, bien sûr, le corps de la mère. Elle repose sur une castration, la perte symbolique du petit phallus de chair...

Toutefois, à considérer l'objet qui est toujours désigné au lieu. de la mort dans les pièces de Gauvreau, on peut se demander s'il a choisi de relever la bonne mort, s'il ne s'est pas trompé de mort. La question est sérieuse. La Reprise, comme de nombreux autres textes, nous fait assister à la mort de la femme, et c'est celle-ci qu'il s'agit de délivrer de l'enfer de la mort sociale où on ne lui permet d'exister qu'à la condition, justement, d'être morte, c'est-à-dire dévitalisée, privée de ses attributs. Dans la pièce, cela se traduit par la destruction de ce qui est considéré par Gauvreau comme le propre du féminin (mais aussi bien du poète authentique): sensibilité extrême, amour vrai, sublime, générosité absolue, compassion...

Le Corps sans larmes, qui sert d'amorce et de toile de fond à $\mathrm{La}$ Reprise, c'est l'histoire d'une femme qu'on a "tuée. en la privant de ses "larmes", de sa faculté de sentir, d'éprouver le malheur et d'y. compatir, d'être capable d'affronter et de ressentir la douleur du deuil:

Weddeg, dit la jeune, je souffre. Je souffre d'une souffrance engourdie. Tu sais, Weddeg, quelle affection me liait à mon petit chat. Eh bien, tout à l'heure, je l'ai étranglé de mes propres mains. Il est mort, j'en ai été désolée, mais aucune larme n'a surgi de mon corps ${ }^{-15}$.

Ce dernier, par amour pour elle, et dans une ultime tentative pour lui rendre ses larmes, va donc se poignarder devant elle. Par sa mort, il lui rend la vie, qui se met alors à couler à flot dans le torrent de ses larmes. La vie n'est ainsi rendue que dans la mort, par la mort de l'être aimé qui peut ainsi être introjecté et permettre à la vie de s'ouvrir au déchirement de la présence, d'être res-suscité par l'appel de l'Autre.

Weddeg, les larmes sont rendues à mon corps. Weddeg, je pleure. Et c'est sur toi que je pleure. Mon amour, tu m'es ravi au moment où la palpitation anime mes chairs! Je suis une cascade qui devient océan. Weddeg, tu me rends par le déchirement la présence ouverte. Je suis ressuscitée, mon cher amour ${ }^{16}$.

Cet amour, surhumain, que Gauvreau a toujours porté à la femme, au point d'en paraître parfois ridicule et de susciter des commentaires aussi lucides que désagréables à entendre - pensons à ceux de Jacques Ferron qui voyait en lui un homme n'ayant pu se séparer de sa «maman* - , répond sans doute à des causes plus générales qui

15. Ibid., p. 1267

16. Ibid., p. 1269. 
tiennent à la position singulière qu'occupe l'homme québécois dans la structure symbolique qui l'accueille: au Québec, à défaut de langue forte - celle d'un père qui occuperait pleinement l'espace symbolique et y serait reconnu -, l'homme est souvent porté à se rabattre sur le corps, sur ce qu'on pourrait appeler la "langue de la mère " (où l'échange ne nécessite pas les mots, le langage), attendant qu'elle suture ce trou béant dans les mailles de son identité, voire qu'elle remplisse tout l'espace de son manque-à-être; en somme, qu'elle soit son phallus... mais dès lors aussi sa tombe! C'est là une tentation permanente, une béance insatiable qui n'a guère été pensée (et qui attend encore, dans le domaine de la littérature, son arpenteur ${ }^{17} \ldots$..). Pour cela il faudrait du père, du père ayant laissé héritage à ses fils!

En littérature, Ferron aurait pu jouer ce rôle (et certains le lui auront fait jouer, notamment Victor-Lévy Beaulieu), mais il semble qu'il aura préféré s'en abstenir, jugeant les temps encore trop incertains, trop peu propices pour risquer une telle position. Pareillement chez Borduas, cette autre figure tutélaire. Ce dernier est bien intervenu auprès de Gauvreau, mais à distance, et sans jamais qu'un pacte soit véritablement scellé entre eux. Certes, dans une lettre envoyée de Paris le 22 décembre 1956, Borduas lance un sérieux avertissement à Gauvreau à qui il reproche son attitude chicanière, sa propension au martyre enfin, la vénération quasi maladive qu'il porte à la femme; mais tout cela est fait de mots lointains, comme si l'histoire elle-même parlait à travers lui, qu'il était enfin mort...

D'ailleurs ma peinture file vers un autre monde plus impersonnel, plus général. Fini, pour moi, les petites bêtises sympathiques. C'est tout l'univers que j'ai besoin de saisir à la grecque, à la romaine, à l'américaine!...

Depuis le voyage en Sicile ma rupture est complète avec le christianisme. Même le Moyen Âge, pour lequel je gardais de la sympathie, a

17. Je pense toutefois au court mais capital récit de Jacques Brault, Agonie (Montréal, Boréal Express, 1985), qui est sans doute celui qui a été le plus loin dans l'expérience et l'apprentissage de la mort dans le rapport à l'autre et à soi-même. Son récit, tout entier placé sous le signe d'une mort désirée, et surtout reconnue comme inaugurale (*Mourir, acte initial plutôt que terminal•) se termine ainsi: «Il se mourait. Moi aussi. Chacun à sa manière. Tous deux ensemble. L'espace d'une minute, nous avons formé un lieu de connivence, un pays. Une promesse? Non. Nous sommes tous des exilés. Nous ne rentrerons pas au pays. Il n'y a pas, il n'y a jamais eu de pays, il n'y aura jamais de pays [...] Le lieu n'est que d'angoisse, une étroitesse, un resserrement d'être. La lutte se donne des raisons d'y croire, d'espérer que la vie triomphera. Mais il est sur son banc, défait, décomposé. Il n'attendait rien d'autre. Je n'irai pas au bureau. Ni sur sa tombe, si on lui en donne une. Je n'irai pas. Je mourrai sans mourir.• 
sauté. Il ne s'agit plus de s'isoler dans l'objet de notre amour, cathédrale ou femme, d'un univers ressenti hostile et douloureux, mais à l'occasion de cet objet de rejoindre la divine impersonnalité - que nous dirions aujourd'hui - cosmique $[. . .]^{18}$.

Cet avertissement, entendons-le comme la réprimande adressée par un père à un fils chez qui il n'a pas encore reconnu l'homme, capable de se donner sans réserve à l'œuvre, c'est-à-dire à la mort, dans la divine impersonnalité qu'exige tout travail de création, qui est incapable de s'abstraire de sa petite histoire, qui se donne toujours comme sujet unique, bouc émissaire du malheur, alors qu'il prétend encore faire preuve de générosité... Devrons-nous rappeler que Borduas s'inscrit ici dans une vieille lignée: celle de ceux qui ont choisi de s'exiler pour enfin vivre, plutôt que de vivre la mort au quotidien dans un pays ingrat et stérile? Il a choisi de tuer la mort dans l'exil, de ne s'en occuper que pour autant qu'elle concernait son œuvre et s'imposait à elle comme sa limite.

Et cela m'amène à poser à Gauvreau, et sans doute à travers lui à une bonne partie de la littérature québécoise, la question suivante: comment le créateur vit-il la mort, quelle figure lui donne-t-il? Mais surtout, quelle langue parle cette mort? Car.la mort n'est-elle pas déjà inscrite, ici, dans le rapport que nous entretenons avec la langue lieu par excellence de l'épreuve symbolique?

Nous vivons, au Québec, dans une incertitude permanente à l'égard des signes, du langage. Trop nombreux sont ceux qui l'ont souligné pour que nous y revenions. En revanche, ce qui peut nous intéresser, c'est la position particulière qu'y occupe le langage exploréen: une langue qui a choisi de désintégrer l'appareil de mort qui pèse sur la langue québécoise, mais en son lieu même, au sein même de la langue qui a cessé de chanter, d'être vivante.

Ce n'est certainement pas un hasard si Gauvreau, y allant de sa théorie sur la langue, considère cette dernière comme une matière inerte, morte, comme l'alphabet auquel il l'assimile: "La matière à façonner est complètement inerte et impersonnelle et d'ailleurs en elle-même invariable: c'est l'alphabet ${ }^{19}$." Ailleurs, commentant la notion de "style " chez un écrivain, il dira encore: "Le STYLE de Stendhal (comme le style de n'importe quel écrivain puissant) est le fruit spontané de la lutte engagée entre le désir-inquiétude de Stendhal [...] et la matière inerte: c'est-à-dire le langage - ou plus

18. Liberté, avril 1962 , p. $239-240$.

19. Correspondance, op. cit., 26 janvier 1950, p. 10. 
précisément: l'alphabet de la langue française ${ }^{20}$. Pas étonnant non plus qu'il prétende que seul le volcan émotif puisse venir à bout de ce formidable barrage qui retient les eaux de notre langue. Une langue qu'on aurait privée de ses larmes ${ }^{21}$ ?

On se rappelle comment Nicolas Abraham ${ }^{22}$ définit la "crypte". C'est le lieu psychique où gisent les "mots enterrés vifs, murés et forclos", mots s'opposant à "l'opération symbolique intégrale" qui consiste à remonter du trauma d'un événement à un autre, de la perte pleurée à la perte ignorée. En ce sens, on peut dire qu'un mort peut toujours en cacher un autre... Cette morte qui revient sans cesse illuminer les eaux de la langue boueuse, désécrire, raturer le texte gauvrien, ne ressemble-t-elle pas à tout ce qui, autres habitants de notre inconscient, a été muré dans la langue: mots interdits, paroles tues, gestes avortés, désirs gelés sur' pied, au pied d'une lettre tonitruante qui, chez Gauvreau, retrouve sa propre autonomie? Et ce que soulève/relève/désensevelit Gauvreau à travers ses glossolalies, n'estce-pas une langue barrée, emmurée vivante, une langue interdite par l'Histoire, par le bilinguisme qui rature la langue maternelle, par l'absence de père?

Sauvage et agressive, bégayante mais articulée avec la plus grande rigueur, blasphématoire mais portant soutane, visant toutes les langues $^{23}$ (de Pentecôte et d'ailleurs) alors qu'une seule est déjà impossible, dans ses glossolalies se mêlent tous les procès de langue(s) qui peuvent hanter un poète, né au Québec, possédé comme le fut Gauvreau par. le démon poétique, et obsédé, comme lui, par la mort.

lazmoitoidloghelanghan ourptf izniglitipiliafauchéghoglolgrégalduzuzhéhométafoilapérouachwouagligd rumina fanferlin ouptin iglou stou plumniumn uculuculiculenculaglumenclumnostrovlidéjéjaujouir

Jappements à la lune, 25 mars 1970

20. Ibid., p. 9.

21. Rappelons que Michel de Certeau voyait une profonde parenté entre le adon des larmes = et le -don des langues * (glossolalie). Cf. son article «Glossolalies: utopies vocales, Traverse, $n^{\circ} 20$, novembre 1980.

22. Cf. Nicolas Abraham et Maria Torok, Le Verbier de l'bomme aux loups, précédé de Jacques Derrida, Fors, Paris, Aubier-Flammarion, 1976.

23. Domitien d'Olmansay - - Le bilinguisme est préférable à l'unilinguisme et toute langue universelle est une possibilité flagrante d'authenticité. (Rose enfer des animaux, (Euvres créatrices complètes, p. 827) 